\title{
Molecular Growth upon Ionization of Van der Waals Clusters Containing HCCH and HCN is a Pathway to Prebiotic Molecules.
}

Tamar Stein ${ }^{1,2 \# @, ~ P a r t h a ~ P . ~ B e r a ~}{ }^{3,4 @}$, Timothy J. Lee ${ }^{3} \&$ Martin Head-Gordon ${ }^{1,2 *}$

1 Chemical Sciences Division, Lawrence Berkeley National Laboratory, Berkeley, CA, 94720, USA

2 Department of Chemistry, University of California, Berkeley, CA, 94720, USA

3 Space Science and Astrobiology Division, NASA Ames Research Center, MS 245-6, Moffett Field, Mountain View, CA 94035, USA

4 Bay Area Environmental Research Institute, NASA Research Park, Moffett Field, CA 94035, USA

Corresponding author E-mail: mhg@ @chem.berkeley.edu

${ }^{\circledR}$ These authors made equal contributions.

\# Present address: Fritz Haber Research Center for Molecular Dynamics, The Hebrew University of Jerusalem, Jerusalem 9190401, Israel 


\begin{abstract}
The growth mechanisms of organic molecules in an ionizing environment such as the interstellar medium are not completely understood. Here we examine by means of ab initio molecular dynamics (AIMD) simulations and density functional theory (DFT) computations the possibility of bond formation and molecular growth upon ionization of Van der Waals clusters of pure HCN clusters, and mixed clusters of $\mathrm{HCN}$ and $\mathrm{HCCH}$, both of which are widespread in the interstellar medium. Ionization of van der Waals clusters can potentially lead to growth in low temperature and low-density environments. Our results show, that upon ionization of the pure HCN clusters, strongly bound stable structures are formed that contain $\mathrm{N} \cdots \mathrm{H}$ bonds, and growth beyond pairwise HCN molecules is seen only in a small percentage of cases. In contrast, mixed clusters, where $\mathrm{HCCH}$ is preferentially ionized over $\mathrm{HCN}$, can grow up to 3 or 4 units long with new carboncarbon and carbon-nitrogen covalent bonds. Moreover, cyclic molecules formed, such as the radical cation of pyridine, which is a prebiotic molecule. The results presented here are significant as they provide a feasible pathway for molecular growth of small organic molecules containing both carbon and nitrogen in cold and relatively denser environments such as in dense molecular clouds but closer to the photo-dissociation regions, and protoplanetary disks. In the mechanism we propose, first, a neutral van der Waals cluster is formed. Once the cluster is formed it can undergo photoionization which leads to chemical reactivity without any reaction barrier.
\end{abstract}




\section{Introduction}

Organic molecules are known to exist in all stages of stellar evolution starting from the diffuse clouds to the protoplanetary disks with a wide variety in their abundance and complexity. Complex organics such as the polycyclic aromatic hydrocarbons (PAHs) are known to be widespread in many environments, and are believed to account for about 20 percent of all carbon in the interstellar medium..$^{1-7}$ Nitrogen and oxygen containing organic molecules such as nitriles and oxides have been identified in the gas phase interstellar medium (ISM) using radio astronomy. Complex biorelevant molecules such as vitamin B3, sugars, amino acids, nucleic acids and other large molecules have been identified in meteorite samples (e.g. Murchison) via laboratory analysis, and have been established as being of extraterrestrial origin..$^{8-15}$ It is extremely challenging to detect specific PAHs in the gas phase using radio astronomy despite the scientific consensus on their presence in the ISM, since they have been identified as a family by their signature vibrational features around 3.2, 6.2 microns in the emission spectra, ${ }^{4-7,16,17}$ and their molecular size, $\sim 50 \mathrm{C}$ atoms, leads to very small rotational constants. ${ }^{18-20}$

Some theories pertaining to the beginning of life suggest an interstellar source of prebiotic molecules that are transported to telluric planets by small solar system bodies such as meteorites and comets. ${ }^{17,21-24}$ Nitrogenous cyclic molecules have special importance for prebiotic chemistry as such molecules can be precursors of nucleobases - the building blocks of life. Such molecules are yet to be identified in the gas phase interstellar medium (ISM). Many nitriles with a terminal $\mathrm{CN}$ moiety, because of their large dipole moment and intense rotational spectrum, have been identified in dense molecular clouds. ${ }^{21,25-29}$ Notably, benzonitrile has been recently been observed from its hyperfine-resolved transitions in emission in giant Taurus molecular cloud TMC-1. ${ }^{21}$ Evidently, understanding the growth mechanism of prebiotic molecules from the basic building blocks at the molecular level is of fundamental interest. Both laboratory experiments and computer simulations are suitable approaches to explore and understand their formation routes.

Some suggested mechanisms are analogous to those involved in the formation of polycyclic aromatic hydrocarbons. ${ }^{30-32}$ One example is a hydrogen abstraction $\mathrm{C}_{2} \mathrm{H}_{2}$ addition (HACA) like mechanism for the formation of nitrogenated PAHs (PANHs) involving pyridyl radical and acetylene at elevated temperatures. ${ }^{33}$ Other mechanisms include a series of reactions between small-unsaturated molecules and radicals, which subsequently result in PANH formation. ${ }^{34,35} \mathrm{HCN}$ molecules are known to have a role in the synthesis of important prebiotic 
molecules including nucleobases and oligopeptides. ${ }^{23,36,37}$ Specifically, they are a potential precursor to PANH molecules. HCN and other nitriles can be found on asteroids, comets, solar system moons, and planets. ${ }^{37,38}$ So much so that HCN polymers have been hypothesized to be responsible for the brown-orange color of Jupiter, ${ }^{39,40}$ and in some of the photochemically generated organics (tholins) that are responsible for the orange haze in Titan's stratosphere. ${ }^{39,41,42}$

It is known from supersonic beam experiments that ionization of acetylene clusters can cause molecular growth leading to formation of benzene radical cation. ${ }^{43}, 44$ Mass spectrometry has been applied to characterize the products resulting from photoionization of acetylene clusters, and peaks with mass corresponding to intact clusters with the formula $\left(\mathrm{C}_{2} \mathrm{H}_{2}\right)_{\mathrm{n}}{ }^{+}$(where $\mathrm{n}$ is an integer), were observed. ${ }^{45}$ In a recent study, we used $a b$ initio molecular dynamics (AIMD) to model the results of those experiments. ${ }^{45}$ Our results showed that chemically bonded molecules can be formed subsequent to ionization of neutral van der Waals clusters. Upon ionization, the clusters are able to spontaneously rearrange, and undergo geometric relaxation. During the relaxation process, intra-cluster ion-molecule reactions take place, resulting in the formation of covalently bonded structures, among them the benzene cation, the basic building block of PAHs. We observed that spectator acetylene molecules in the clusters can modulate the potential energy surface (PES) such that structures like the cyclobutadiene radical cation can form without any barrier in the cluster environment. Moreover, the spectator acetylene molecules can stabilize the resulting structures by evaporation, taking excess kinetic energy away with them. These reactions can be important in dense molecular clouds where the number density is comparatively higher, the temperature is low, and acetylene is observed. ${ }^{46-49}$ Ionization is often achieved in dense clouds via a mechanism driven by UV rays generated by the de-excitation of $\mathrm{H}_{2}$ following the impact of cosmic ray produced electrons via the so called Prasad-Tarafdar mechanism. ${ }^{50}$ This pathway may contribute to the formation of nitrogenated heterocycles (and smaller precursors) in protoplanetary disks (PPDs). A gas-grain model of protoplanetary disks predicted significant abundance of $\mathrm{HCN}$ and $\mathrm{HCCH}$ in the midplane beyond 100AU. ${ }^{51}$ Another astrophysical environment suitable for large molecular cluster formation is Titan's upper atmosphere where neutral and ionic species are observed by the CASSINI mission. ${ }^{52}$ If $\mathrm{HCCH}$ and HCN neutral cluster for in the ionosphere or Titan and get ionized by colliding with high-energy and oxidizing particles then the mechanism of carbon-nitrogen molecular growth proposed here will be very 
important in Titan's atmosphere. Molecular growth from dimers of acetylene and higher annulenes have indeed been proposed for Titan's atmosphere. ${ }^{53}$

In this manuscript, we present an AIMD and quantum chemistry study of the formation routes of different nitrogenated cyclic molecules starting from clusters of pure $\mathrm{HCN}$, and mixed clusters of $\mathrm{HCN}$ and $\mathrm{HCCH}$, upon ionization. Our results will address whether an analogous mechanism can account for the formation of the radical cations of the basic building blocks of life, namely pyridine, pyrimidine, and triazine, in a fashion similar to the formation of benzene radical cation previously seen in a joint laboratory and theoretical study. The remainder of the paper is organized as follows. After discussing computational details, the most stable structures of neutral clusters (both pure $\mathrm{HCN}$ and mixed $\mathrm{HCN}$ and $\mathrm{C}_{2} \mathrm{H}_{2}$ ), ranging in size from dimers through pentamers are reported. These structures are used as the starting point for AIMD trajectories which model the reaction dynamics after photoionization. The products are characterized, and key intermediates are identified on the underlying potential energy surfaces on which the reactive dynamics has occurred.

\section{Computational Details}

All calculations were performed using the Q-Chem 5 software package. ${ }^{54}$ Structures and relative energies of neutral and ionized clusters were calculated using density functional theory (DFT). Neutral clusters and ionized structures were optimized using the $\omega \mathrm{B} 97 \mathrm{X}-\mathrm{V}$ functional ${ }^{55}$ with the cc-pVTZ basis set. ${ }^{56} \omega \mathrm{B} 97 \mathrm{X}-\mathrm{V}$ is a range separated functional that includes the VV10 dispersion correction, ${ }^{57}$ and as such, is suitable for treatment of both the radical cations and the neutral cluster. Furthermore, in extensive testing, by Goerigk et a ${ }^{58}$ and Mardirossian and HeadGordon, ${ }^{59} \omega \mathrm{B} 97 \mathrm{X}-\mathrm{V}$ was identified as the most accurate hybrid GGA functional both for nonbonded interactions, and overall.

To reduce computational expense, AIMD simulations were performed using the less expensive $\omega \mathrm{B} 97$ functional ${ }^{60}$ with a smaller 6-31g* basis set. Each of the neutral geometries served as starting points for the AIMD simulations, which are propagated on the cation surface to model the post-ionization dynamics. The time step for the AIMD simulations was $1.21 \mathrm{fs}$ (50 a.u) and the total simulation time was $2.4 \mathrm{ps}$ (2000 time-steps). For each starting geometry, 30 trajectories were performed with nuclear velocities initiated to correspond to temperatures between $30 \mathrm{~K}$ and $90 \mathrm{~K}$. 
This temperature range is believed to be appropriate for dense molecular clouds where the number density of particles is relatively high compared to the conditions in a diffuse molecular cloud. 


\section{Results}

\section{III.a Optimized structures of neutral clusters.}

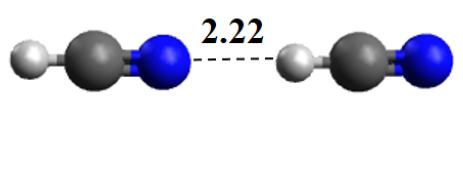

$-4.97 \mathrm{kcal} / \mathrm{mol}$

(a)

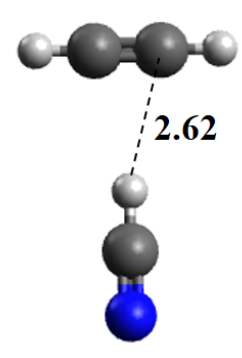

$-2.67 \mathrm{kcal} / \mathrm{mol}$

(b)

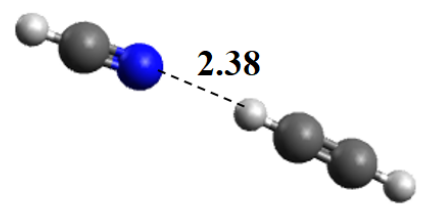

$-2.63 \mathrm{kcal} / \mathrm{mol}$

(c)

Figure 1: Structure of pure $\mathrm{HCN}$ dimer (a) and mixed dimers of $\mathrm{HCCH}$ and $\mathrm{HCN}$ structures $(\mathrm{b}, \mathrm{c})$. Binding energies are given in $\mathrm{kcal} / \mathrm{mol}$ and distances in $\AA$.

To understand the interactions and reactions that can occur upon ionization of HCN clusters (both pure and when mixed with acetylene), we started by studying the geometry of the neutral clusters. For each cluster, we calculated the binding energy as the difference between the electronic energies of the cluster and the separated molecules. First, we studied the dimer structures; $(\mathrm{HCN})_{2}$ is shown in Figure 1(a), and structures for $\mathrm{HCN} \cdots \mathrm{C}_{2} \mathrm{H}_{2}$ are given in Figure 1(b, c). For $(\mathrm{HCN})_{2}$, the hydrogen of one $\mathrm{HCN}$ is pointed towards the nitrogen of the second $\mathrm{HCN}$ molecule; the dimer has a typical hydrogen-bond with a binding energy of $-4.97 \mathrm{kcal} / \mathrm{mol}$ (Figure 1(a)). For $\mathrm{HCN} \cdots \mathrm{C}_{2} \mathrm{H}_{2}$, two configurations that are very close in energy were found, and are shown in Figure 1(b, c). A T-shaped structure between the hydrogen of the HCN and the $\pi$ cloud of the acetylene molecule is found to be lower in energy, with binding energy of $2.67 \mathrm{kcal} / \mathrm{mol}$. The second configuration is linear $(\mathrm{C} \propto \mathrm{V})$, where the $\mathrm{N}$ atom of the $\mathrm{HCN}$ is pointing towards the $\mathrm{H}$ atom of the acetylene, as shown in Fig 1 (c), with a binding energy of $2.63 \mathrm{kcal} / \mathrm{mol}$. Due to the large dipole moment (calculated as $3.02 \mathrm{D})$ of $\mathrm{HCN}$ relative to acetylene $(0 \mathrm{D})$, the binding energy of $(\mathrm{HCN})_{2}$ is almost twice that of $\mathrm{HCN} \cdots \mathrm{C}_{2} \mathrm{H}_{2}$. Because of the dipole-charge interaction (in the T-shaped isomer) and dipole-induced dipole interaction (in the linear isomer), the $\mathrm{HCN} \cdots \mathrm{C}_{2} \mathrm{H}_{2}$ structures are more strongly bound than the acetylene dimer, which has binding energies of 1.6 and $1.4 \mathrm{kcal} / \mathrm{mol}$ for the T-shaped and the slipped parallel structures, respectively. ${ }^{35,43}$ 


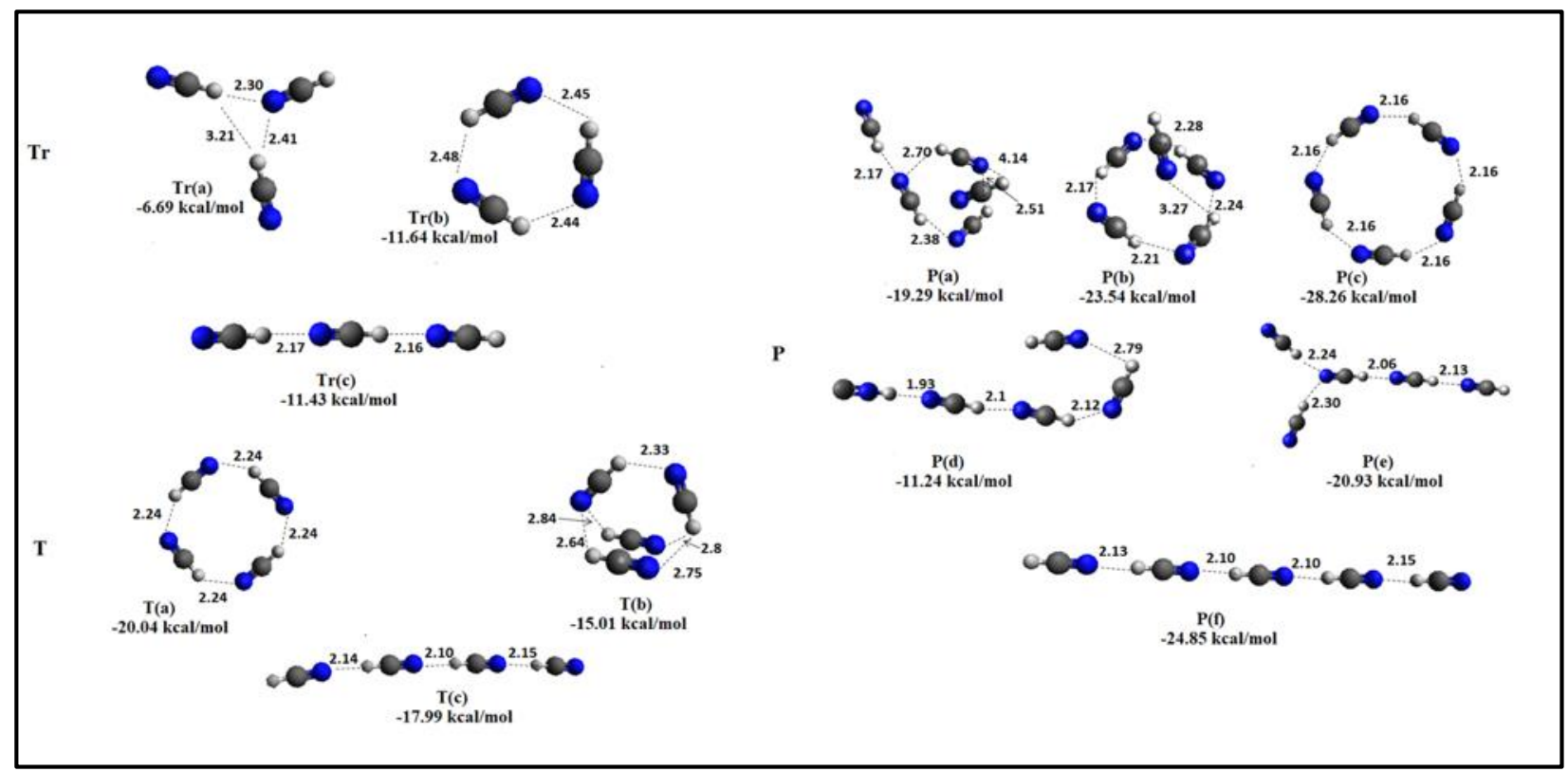

Figure 2: Structures of pure HCN Trimer (Tr), Tetramer (T) and Pentamer (P) clusters. Binding energies are given in kcal/mol and distances in $\AA$.

Optimized structures for larger $(\mathrm{HCN})_{n}$ clusters, specifically the trimer, tetramer and pentamer, are shown in Figure 2. These structures are quite interesting and reflect the different ways in which strong dipole-dipole interactions between different HCN molecules can be attained. A head-to-tail orientation of all dipoles leads to linear structures. Bent head-to-tail orientation leads to cyclic (and intermediate) structures that nonetheless feature at least one additional nearestneighbor dipole-dipole interaction. For the trimer, the cyclic and linear configurations are close in energy, with the cyclic structure being more stable due to three (partial) hydrogen-bonding interactions instead of two for linear. The structure in which two HCN molecules have dipoles approaching a head-to-head arrangement, Fig $2 \operatorname{Tr}(a)$, is significantly less strongly bound than the other two structures. Alternatively, and essentially equivalently, one can view this structure as one in which one nitrogen is shared by two $\mathrm{HCN}$ via two weak $\mathrm{N} \cdots \mathrm{H}$ hydrogen bonds. In the larger clusters, namely the tetramer and pentamer, the most stable configurations are again the cyclic the most stable, and the linear - the second most stable. The difference in stability between these two configurations becomes more pronounced in the larger clusters as the cyclic structure is more stabilized than the linear one because of the presence of one additional $\mathrm{N} \cdots \mathrm{H}$ hydrogen bond. Additional cage-like and branched neutral structures were found for tetramer and pentamers clusters. 
We further studied larger trimer, tetramer and pentamer clusters for the mixed $\mathrm{HCCH} / \mathrm{HCN}$ clusters. The structural trends are broadly similar to the trends seen in the dimer case, although other rich details such as weaker, secondary interactions involving the pi-electrons of acetylene interacting with the $\mathrm{H}$ of $\mathrm{HCN}$, and weak $\mathrm{HCCH} \cdots \mathrm{NCH}$ interactions are also evident. The structures of the mixed $\mathrm{HCCH} / \mathrm{HCN}$ clusters are available in Fig X1 - Fig X6 in the supplementary materials. More structural isomers were found in the lower parts of the potential energy surface for the mixed $\mathrm{HCCH} / \mathrm{HCN}$ clusters. These structures include linear, branched, caged, and cyclic isomers. In each case the cyclic structures that maximize the bonding interactions are the lowest in energy. These neutral cluster geometries were used as the starting geometries for optimization following an ionization event, after which radical ions are created at neutral geometries that may be far from their equilibrium radical structures.

\section{III.b Optimized structures of ionized clusters.}

Upon ionization, the clusters undergo structural relexation. Figure 3 shows the structue of the dimer clusters after optimization on the cation surface, using the nuetral geometries as the starting point in the calculations. Figure 3(a) shows the result for pure HCN dimer. Interestingly, we see that the result is a distonic structure, such that the ionized clusters contains $\mathrm{HCNH}^{+}$and $\mathrm{CN}$ radical moieties. According to Mulliken analysis, 0.98 (a.u) of the spin is located on the $\mathrm{CN}$ part, while 0.82 (a.u) of the charge is on the $\mathrm{H}_{2} \mathrm{CN}^{+}$part, as is also supported by the spin density of structure 3(a) which is shown in Figure 4. Binding energies are also reported in Figure 3, and were calculated as the electronic energy difference between the resulting optimized strucutre, and the separated moieties (ionized and neutral HCN structures for the case of 3(a), or ionized acetylene and neutral HCN for the case of 3(b) and 3(c)).

In the case of the mixed clusters, we see that the resulting structures are either the covalently bonded, 3(b), or a much weaker, electrostatically-bonded cluster structure, 3(c). The distance between the acetylene and the $\mathrm{HCN}$ in the cluster structure, $3(\mathrm{c})$, is $1.73 \AA$, shorter than for the neutral cluster (2.38 $\AA$ ), as is expected for a charged cluster. All of these structures exhibit strong enough binding energies to survive in low-density cold environments, if they can dissipate the excess kinetic energy with which they will be formed. 


\subsection{4

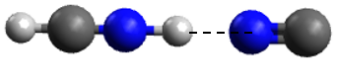

$-55.98 \mathrm{kcal} / \mathrm{mol}$

(a)

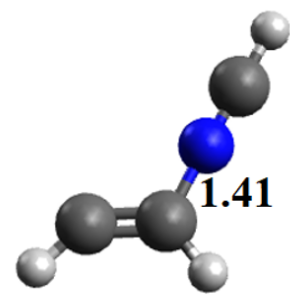

$-53.87 \mathrm{kcal} / \mathrm{mol}$

(b)

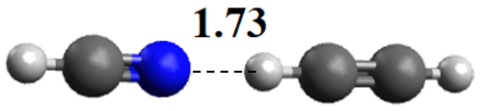

$-20.74 \mathrm{kcal} / \mathrm{mol}$

(c)

Figure 3: Optimized radical cation structures starting from neutral geometries shown in Figure 2. (a) Starting from pure HCN dimer, (b) and (c) starting from mixed acetylene and HCN dimer. BE are given in $\mathrm{kcal} / \mathrm{mol}$ and distances in $\AA$. Note the barrierless formation of a $\mathrm{CN}$ bond in (b).

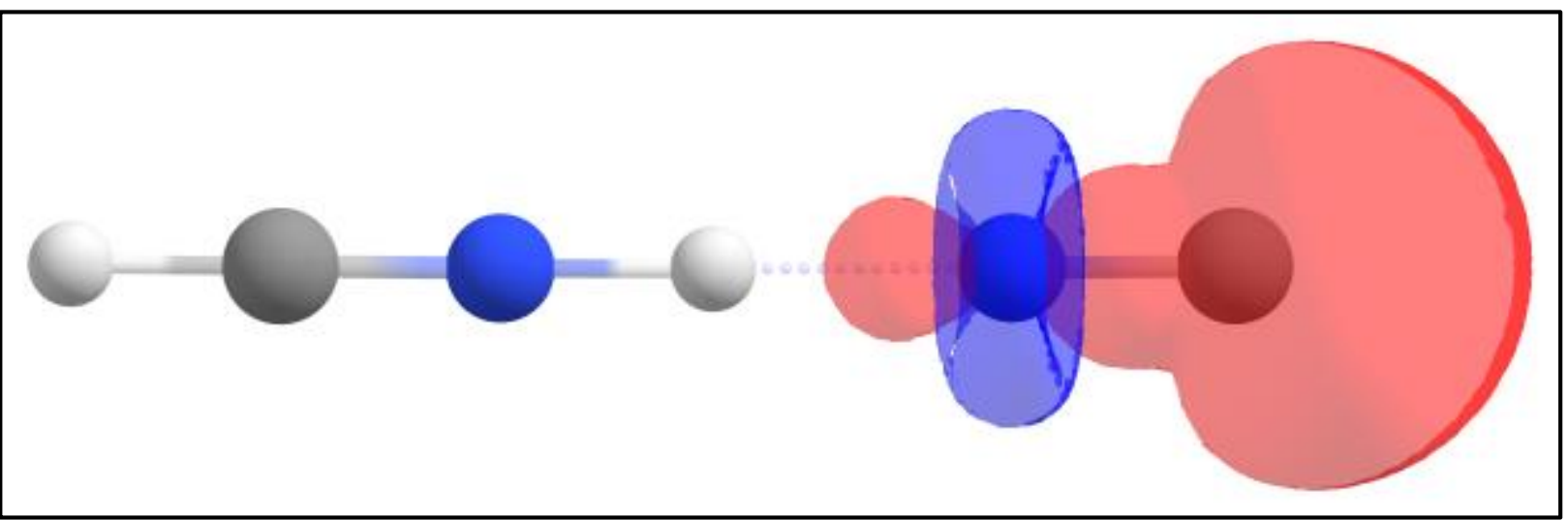

Figure 4: Spin density of $\mathrm{CN}^{-} \mathrm{H}_{2} \mathrm{CN}^{+}$complex, corresponding to structure (a) of Figure 3, showing that this distonic complex consists of protonated $\mathrm{HCN}$ (closed shell), and the neutral $\mathrm{CN}$ radical.

\section{III.c Ab initio molecular dynamics studies of ionized $(\mathrm{HCN})_{n}$ clusters.}

Trajectories were run for pure HCN trimer, tetramer, and pentamer clusters on the cationic surface.

Figure 5 presents the distribution of the structures at the end of the simulations of pure HCN trimer, tetramer and pentamer cationic clusters (total number of trajectories is 179). As shown in Figure 5, the majority of the simulations result either in H-transfer, such that the ionized clusters produce a protonated $\mathrm{HCN}\left(\mathrm{HCNH}^{+}\right)$and $\mathrm{CN}$ radical moieties $(63 \%, 34 \%$ and $84 \%$ for the trimer, tetramer, and pentamer, respectively), or a bonded $\mathrm{C}_{2} \mathrm{~N}_{2} \mathrm{H}_{2}{ }^{+}$structure $(34 \%, 56 \%$ and $12 \%$ for the trimer, tetramer and pentamer, respectively). To better understand the resulting core structures, we optimized the cation structures starting from the pure trimer structures, as shown in Figure 6. At the bottom of the figure the $(\mathrm{HCN})^{+}{ }_{2}$ structure which is a result of bonding between two $\mathrm{HCN}$ units is shown, and at the top of Figure 6, the H-transferred structure is shown. The binding energies of 
the structures are given and as before, calculated as the electronic energy differences between the optimized structure, and the separated HCN molecules (in the case of the trimer, two neutrals and one ionized). Optimizxation starting from the tetramer and pentamer clusters results in similar core structures as shown in Figures 7 and 8 (with additional spectator HCN units). Looking at Figures 6-8, we can identify two key structures, a distonic $\mathrm{C}_{2} \mathrm{H}_{2} \mathrm{~N}_{2}{ }^{+}$structure complexed to the rest of the HCN molecules, which was also observed in the dimer cation case, and a covalently bonded $\mathrm{C}_{2} \mathrm{H}_{2} \mathrm{~N}_{2}{ }^{+}$structure. We calculated the binding energy of $\mathrm{C}_{2} \mathrm{H}_{2} \mathrm{~N}_{2}{ }^{+}$structure with respect to two HCN moieties to compare with the binding energies of Figure 3. The calculated binding energy of the structure is $-78.24 \mathrm{kcal} / \mathrm{mol}$, which demonstrates the stability of this structure. Mulliken analysis indicates that the spin is located on the central nitrogen atom.

The high stability of the $\mathrm{C}_{2} \mathrm{H}_{2} \mathrm{~N}_{2}{ }^{+}$structure is probably the reason that bonding between more than two units upon ionization was observed only at small percentage of the trajectories. Figure 5 shows "Other" structures that are formed in very low percentages and mostly involved a bond between two HCN units along with an H-transfer. We did not observe the formation of triazine cation, or any other cyclic structure; however, $1 \%$ of the trajectories in the tetramer clusters resulted in a bonded ( $\mathrm{C}-\mathrm{N}$ covalent bond) open $\mathrm{C}_{3} \mathrm{~N}_{3} \mathrm{H}_{3}{ }^{+}$structure, shown in Figure 9, which might lead to triazine if allowed to evolve for a longer time. The fact that we do not observe formation of triazine could be due to limited propagation time in the simulation (our typical trajectory length of a few picoseconds is set by computer resource limits). In a previous study, we studied pure acetylene clusters and demonstrated that they can lead to molecular growth; all the trajectories in the acetylene case (for trimer, tetramer and pentamer clusters) led to a covalently bonded structure, and the bonded structure could include more than two acetylene units (e.g. benzene cation). ${ }^{45}$ In contrast, in the pure $\mathrm{HCN}$ case, a large number of the trajectories (as can be seen from Figure 5) ended up in a cluster in which a proton transfer occurs, and bonding between more than two HCN units was only observed in a small number of trajectories. We attribute this to the fact that the newly formed structures are strongly bound, as evident from their large binding energies. Analysis of the electron density difference upon ionization of one unit of acetylene or HCN reveal that in both cases the hole is in the pi-space. However, it is possible that nitrogen radical is less effective in attacking other units than carbon radical as in the acetylene case. 


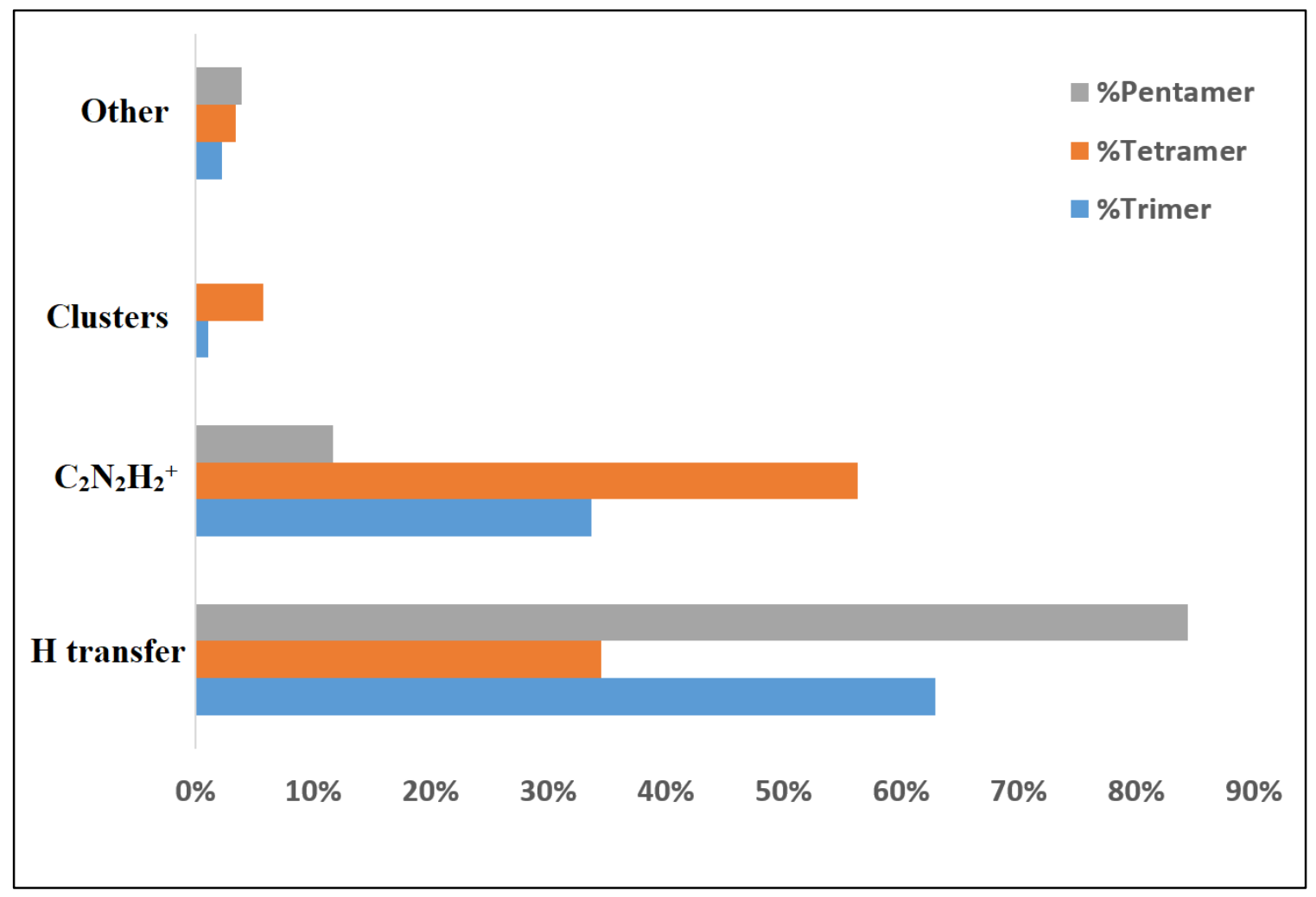

Figure 5: Distribution of resulting structures at the end of the pure clusters' simulations. Blue: structures that start from pure trimer clusters, Orange: structures that starts form pure tetramer structures and Gray: structures that start from pure pentamer clusters. 


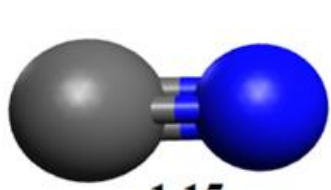

1.15
1.65

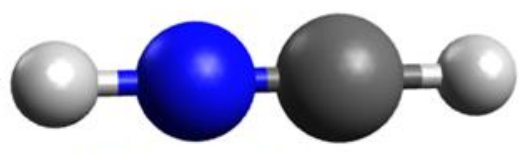

1.06

1.13

1.12

1.74

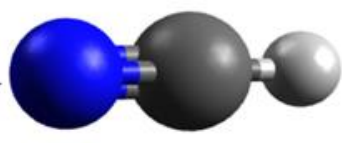

1.14

1.07

(a)

$-75.29 \mathrm{kcal} / \mathrm{mol}$

(b)

$-95.74$

$\mathrm{kcal} / \mathrm{mol}$

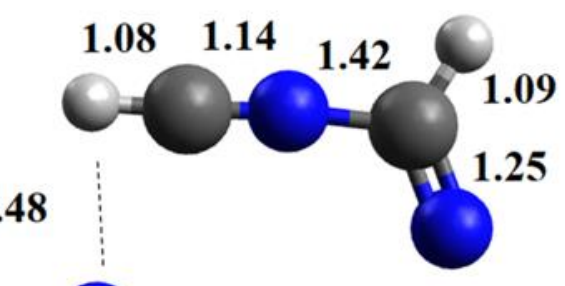

Figure 6: Structures of $\mathrm{C}_{2} \mathrm{H}_{2} \mathrm{~N}_{2}{ }^{+}$(bottom) and the proton-transfer cation (top) clusters that are obtained by optimizing the trimer cluster on the cation surface. BE are given in $\mathrm{kcal} / \mathrm{mol}$ and distances are given in $\AA$.

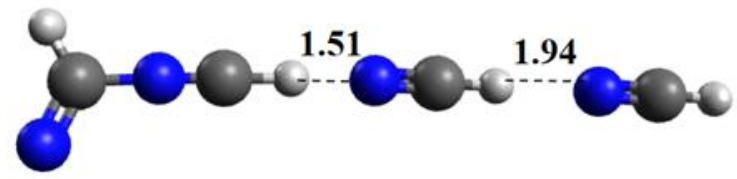

(a)

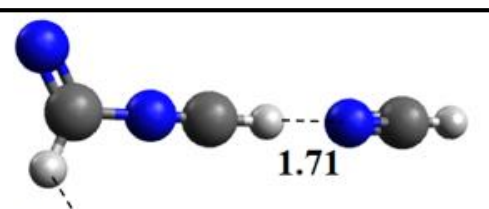

2.35

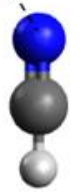

(b)

$-113.54 \mathrm{kcal} / \mathrm{mol}$

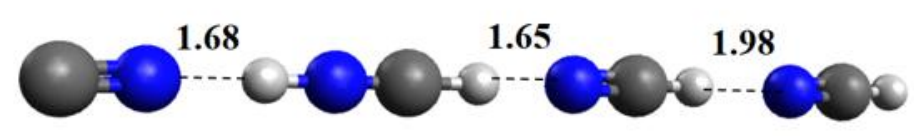

(c)

$-86.88 \mathrm{kcal} / \mathrm{mol}$

Figure 7: Structures obtained by optimized the tetramer clusters on the cation surface. BE are given in kcal/mol and distances in $\AA$. 


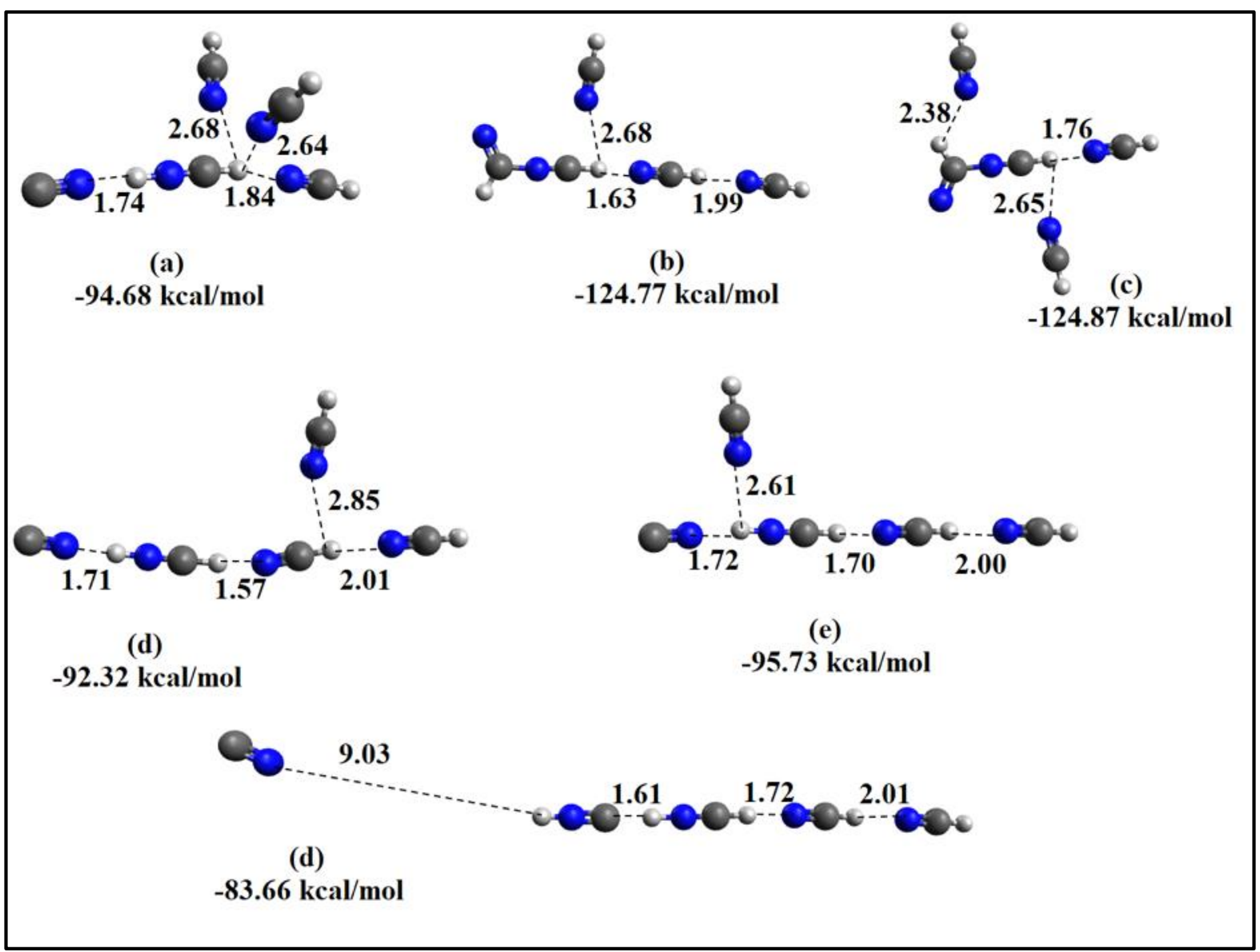

Figure 8: Structures obtained by optimization of the pentamer clusters on the cation surface. BE are given in kcal/mol and distances in $\AA$.

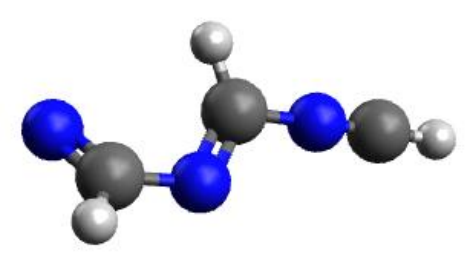

Figure 9: $\mathrm{C}_{3} \mathrm{H}_{3} \mathrm{~N}_{3}{ }^{+}$structure after re-optimization.

Doping the HCN clusters with acetylene molecules might lead to better growth upon ionization, as in the mixed structures, the acetylene, which has a lower ionization potential is expected to be ionized. We therefore studied these mixed $\mathrm{HCN}$ and acetylene clusters as discussed in the next section.

III.d Ab initio molecular dynamics for ionized $(\mathrm{HCN})_{2}\left(\mathrm{C}_{2} \mathrm{H}_{2}\right)_{x}$ clusters. 
We start by studying clusters containing $2 \mathrm{HCN}$ units and $x \mathrm{HCCH}$ units (where $x=1,2,3$ is the number of acetylene molecules; ratio of HCN:acetylene for trimer, tetramer and pentamer is 2:1, $2: 2$ and $2: 3$, respectively). The total number of trajectories are 89,383 , and 240 for the trimer, tetramer and pentamer, respectively.

As suspected, mixed clusters containing both acetylene and HCN units lead to growth beyond two units and result in larger bonded structures, as shown in Fig 10. Upon ionization of neutral mixed $2 \mathrm{HCN}: x \mathrm{HCCH}$ structures, $36 \%$ and $48 \%$ of the tetramer and pentamer trajectories led to bonded $\mathrm{C}_{4} \mathrm{H}_{4}{ }^{+}$, respectively. Additionally, $30 \%, 36 \%$ and $33 \%$ of the trimer, tetramer and pentamer trajectories (respectively) produced covalently bonded $\mathrm{C}_{3} \mathrm{H}_{3} \mathrm{~N}^{+}$that is a direct association product of one $\mathrm{HCN}$ and $\mathrm{HCCH}^{+}$. Larger bonded structures were also observed. For example, $10 \%$ of the pentamer trajectories resulted in the $\mathrm{C}_{6} \mathrm{H}_{6}{ }^{+}$structure, and $7 \%$ and $8 \%$ of the tetramer and pentamer trajectories ended up with $\mathrm{C}_{5} \mathrm{H}_{5} \mathrm{~N}^{+}$structures. These results are potentially very significant as they demonstrate a growth mechanism for larger nitrogen containing compounds from neutral clusters of acetylene and HCN, both of which are widespread in the ISM and other astrophysical environments such as protoplanetary disks (PPDs).

Among the $\mathrm{C}_{5} \mathrm{H}_{5} \mathrm{~N}^{+}$structures, the radical cation of pyridine, a cyclic molecule with nitrogen in the ring, was also found. Although most of the trajectories that ended in $\mathrm{C}_{5} \mathrm{H}_{5} \mathrm{~N}^{+}$did not end up with pyridine, some did. This could be due to the limited propagation time of the simulation. With longer propagation time, some of the intermediate structures are likely to end up as pyridine. For a small percentage $(1 \%)$ of the pentamer trajectories, even larger moieties were formed, such as $\mathrm{C}_{7} \mathrm{H}_{7} \mathrm{~N}^{+}$which is built from three acetylene units and one $\mathrm{HCN}$ unit. Some pentamer trajectories also produced $\mathrm{C}_{6} \mathrm{H}_{6}{ }^{+}$. 


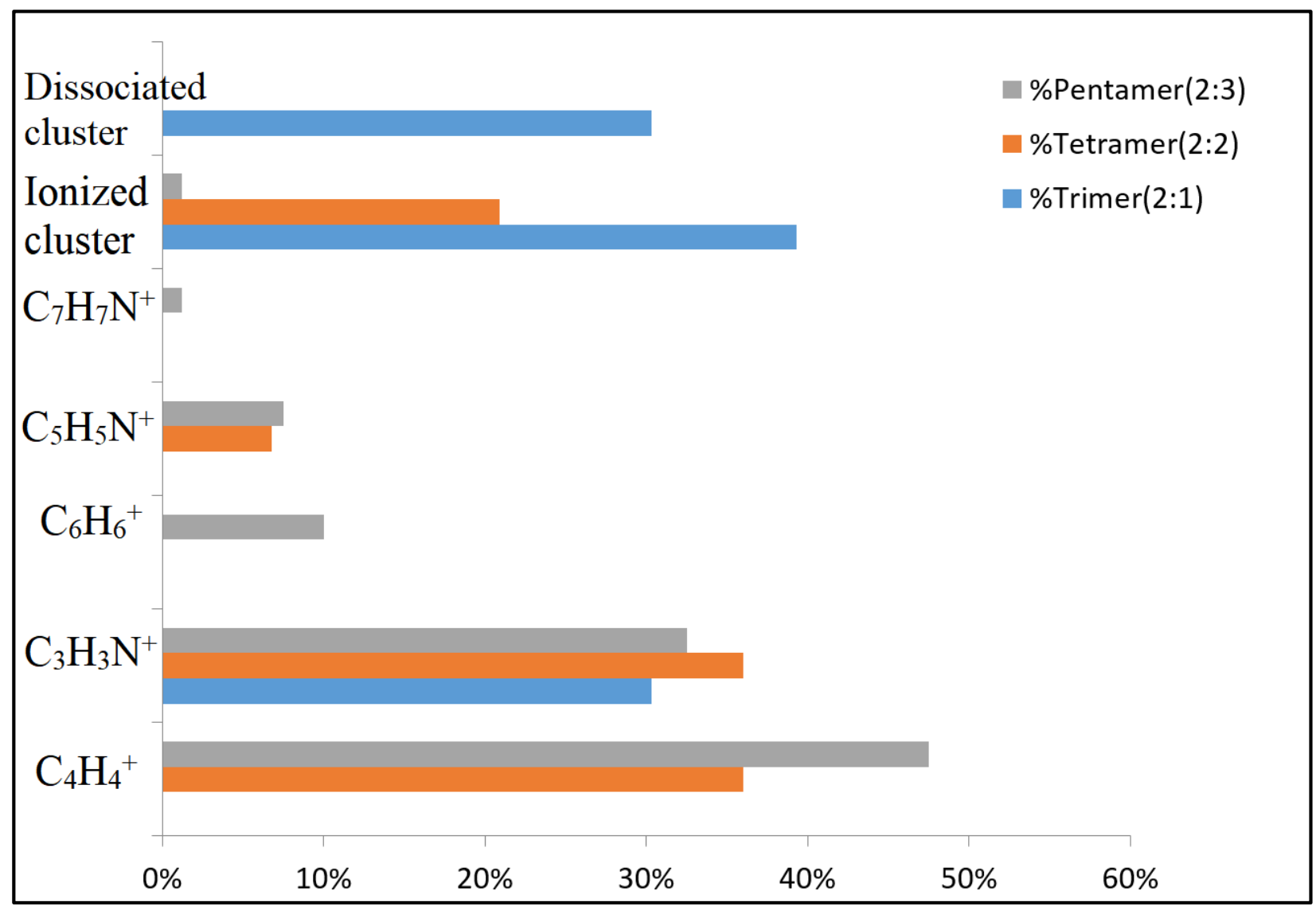

Figure10: Distribution of resulting structures at the end of the mixed (2:x) cluster simulations. Blue: structures that start from (2:1) trimer clusters, Orange: structures that start from (2:2) tetramer structures and Gray: structures that start from (2:3) pentamer clusters.

For the trimer case, $30 \%$ of the clusters dissociate as the trajectories unfold, and at the end of the trajectory run, we observe three molecules at large distance from one another ("dissociated cluster" in Figure 10). Additionally, some of the trajectories did not end up with a covalently bonded structure but with non-covalently bonded clusters. $39 \%$ of the trimer, $21 \%$ tetramer, and $1 \%$ pentamer trajectories end up in non-covalently bonded clusters. As the cluster size grows the clusters tend to make more covalently-bonded structures rather than staying in a cluster. This is possibly because of better vibrational energy dissipation after covalent bond formation in the larger clusters compared to the smaller clusters. The results demonstrate that mixed clusters with $2 \mathrm{HCN}$ and $X$ acetylene units can lead to growth of larger molecules compared to the pure HCN clusters, which might indicate the effectiveness of electrophilic attack of ionized acetylene in attacking neighboring nucleophilic HCN molecules. 
Figure 11 presents the different isomers we get for the core structures with stoichiometries $\mathrm{C}_{3} \mathrm{H}_{3} \mathrm{~N}^{+}, \mathrm{C}_{5} \mathrm{H}_{5} \mathrm{~N}^{+}$and $\mathrm{C}_{7} \mathrm{H}_{7} \mathrm{~N}^{+}$, after we optimize them. As one can see from the figure, we get one $\mathrm{C}_{3} \mathrm{H}_{3} \mathrm{~N}^{+}$isomer, which we discussed above. We get seven isomers of $\mathrm{C}_{5} \mathrm{H}_{5} \mathrm{~N}^{+}$, including the pyridine cation. Two of the structures ( $\mathrm{b}$ and $\mathrm{g}$ ) are likely to lead to pyridine and are analogs of the intermediate structures on the $\mathrm{C}_{6} \mathrm{H}_{6}{ }^{+} \mathrm{PES}$, that can lead to the benzene cation. ${ }^{45}$ The rest of the structures are non-cyclic, and two of them contain hydrogen transfer (e and f). Interestingly, the structures that we get on the $\mathrm{C}_{7} \mathrm{H}_{7} \mathrm{~N}^{+} \mathrm{PES}$ are all cyclic. Structure (a) contains a five-membered ring bonded to an additional chain, and structure (c) is pyridine based. Structure (b) on the $\mathrm{C}_{7} \mathrm{H}_{7} \mathrm{~N}^{+}$ PES is a large ring containing both carbon and nitrogen; however, this structure is very rare (seen only in one trajectory).

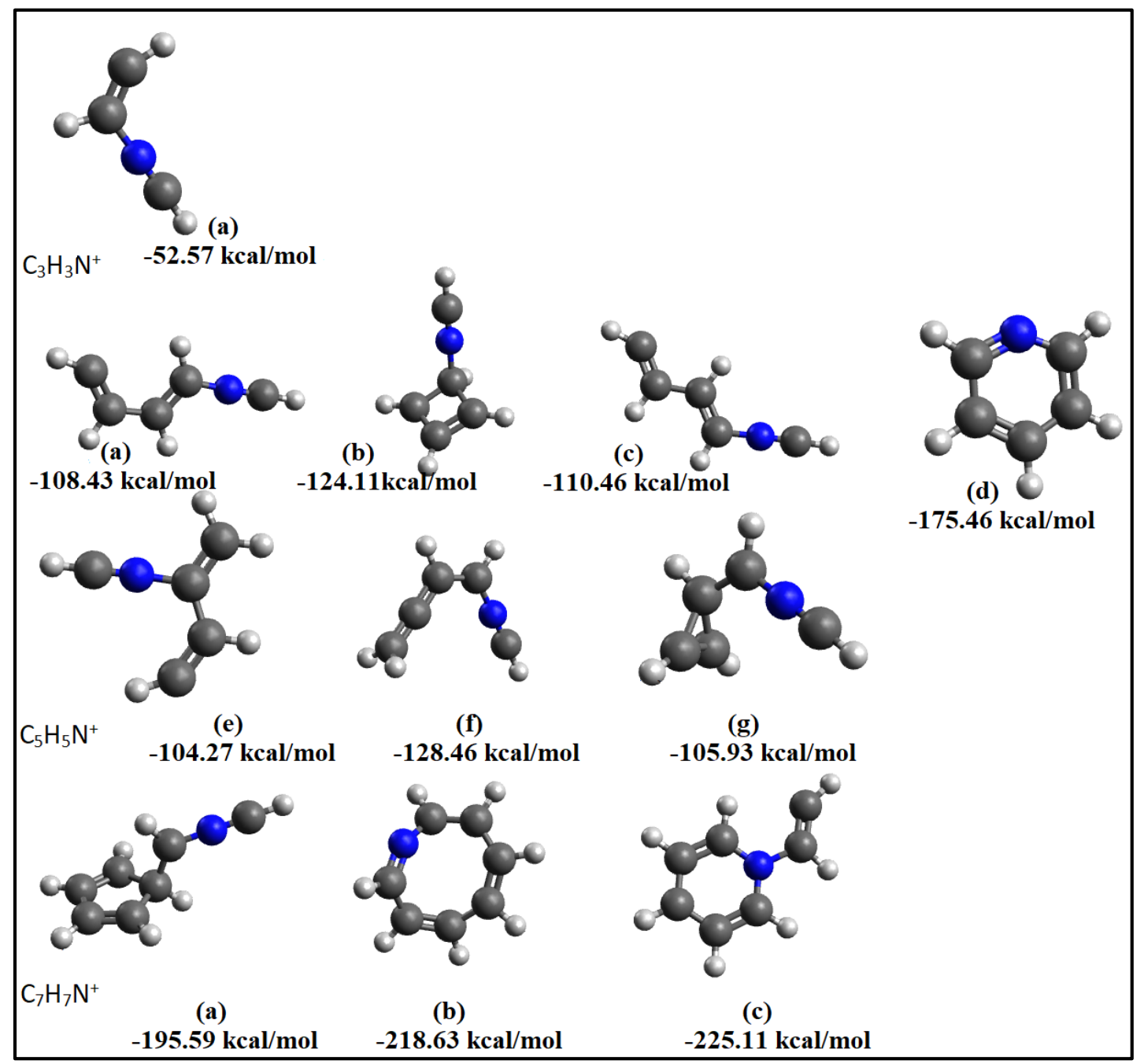

Figure 11: Different cores structures (after re-optimization) obtained in the MD simulations starting from ionization of optimized neutral $(\mathrm{HCN})_{2}\left(\mathrm{C}_{2} \mathrm{H}_{2}\right)_{x}(x=1,2,3)$ structures. Binding energies are calculated with respect to the building blocks: $\mathrm{HCN}$ and $\mathrm{HCCH}^{+}$for $\mathrm{C}_{3} \mathrm{H}_{3} \mathrm{~N}^{+}$structure, $\mathrm{HCN}, \mathrm{HCCH}$ and $\mathrm{HCCH}$ for the case of $\mathrm{C}_{5} \mathrm{H}_{5} \mathrm{~N}^{+}$structures and $\mathrm{HCN}$, two $\mathrm{HCCH}$ and $\mathrm{HCCH}^{+}$for the case of $\mathrm{C}_{7} \mathrm{H}_{7} \mathrm{~N}^{+}$structures. 


\section{III.e Ab initio molecular dynamics for ionized $(\mathrm{HCN})\left(\mathrm{C}_{2} \mathrm{H}_{2}\right)_{x}$ clusters.}

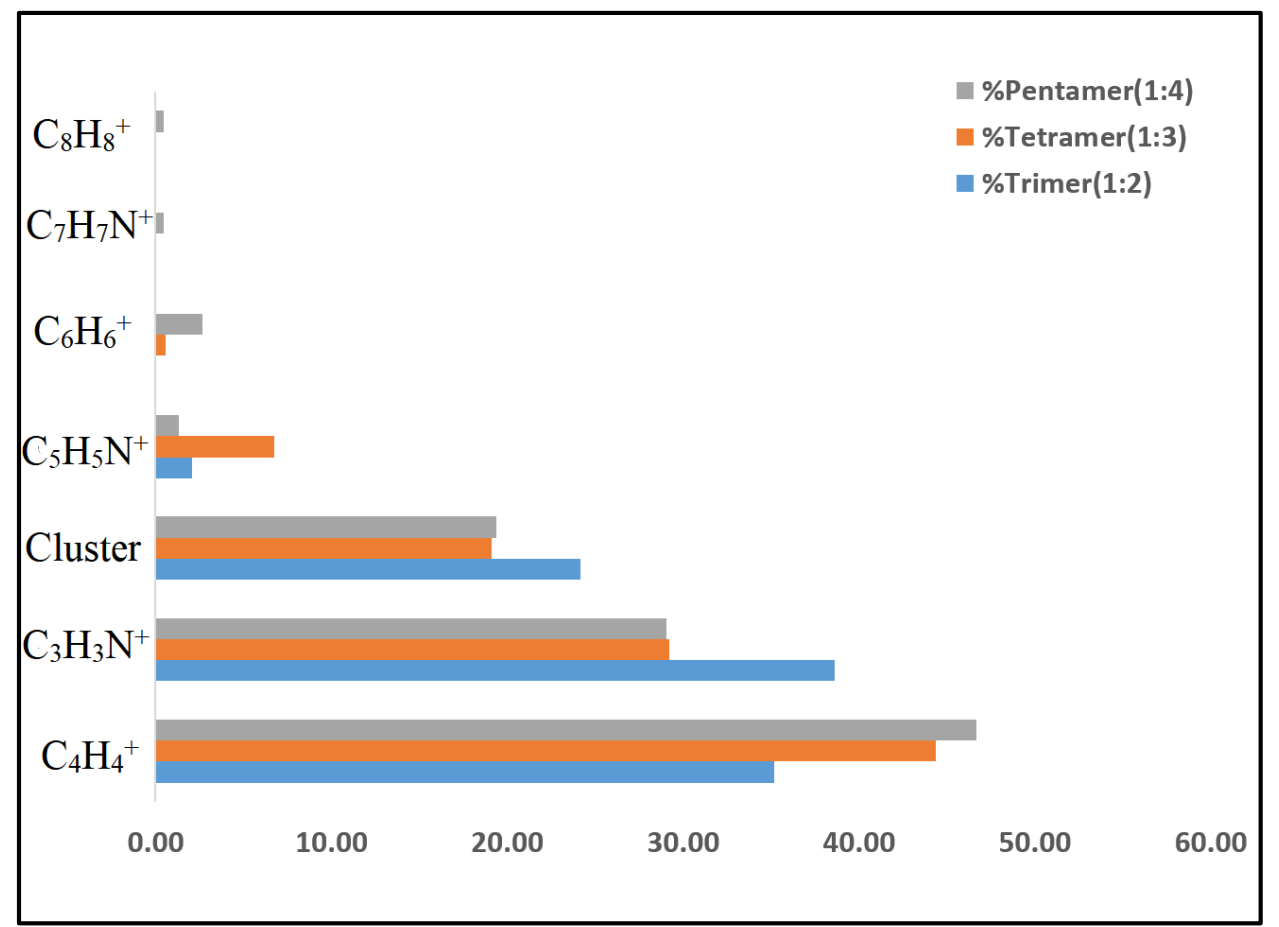

Figure 12: Distribution of resulting structures at the end of the mixed (1:x) cluster simulations. Blue: structures that start from (1:2) trimer clusters, Orange: structures that starts from (1:3) tetramer structures and Gray: structures that start from (1:4) pentamer clusters.

Next, we analyze the results of AIMD simulations for $1 \mathrm{HCN}$ with 2, 3 and 4 acetylenes as shown in Figure 12. The ratio of $\mathrm{HCN}: \mathrm{HCCH}$ is $1: 2,1: 3$ and 1:4 with total number of trajectories of 140, 144 and 183 for trimer, tetramer and pentamer, respectively. Here trimer refers to the ions of $1 \mathrm{HCN}: 2 \mathrm{HCCH}$, tetramer refers to the ions of $1 \mathrm{HCN}: 3 \mathrm{HCCH}$, and pentamer refers to the ions of $1 \mathrm{HCN}: 4 \mathrm{HCCH}$ neutral clusters.

The $1 \mathrm{HCN}: x \mathrm{HCCH}$ clusters behave in a similar manner to $2 \mathrm{HCN}: x \mathrm{HCCH}$ clusters, but with important differences. For the trimer about 25\%, and for tetramer and pentamers about $20 \%$ of the trajectories end up as non-covalently bonded clusters (Figure 12). Most dominant of the product outcome is $\mathrm{C}_{4} \mathrm{H}_{4}{ }^{+} .35 \%$ of the trimers, $45 \%$ of the tetramers, and $48 \%$ of the pentamers form a stable $\mathrm{C}_{4} \mathrm{H}_{4}{ }^{+}$core, and remaining $\mathrm{HCN}$ or $\mathrm{HCCH}$ either stay non-covalently bound to it or dissociate completely. Diluting the $\mathrm{HCN}$ in the system, i.e. having more $\mathrm{HCCH}$ compared to HCN, tend to give more $\mathrm{C}_{4} \mathrm{H}_{4}{ }^{+}$, which is purely carbonaceous. Diluting the $\mathrm{HCN}$ in the system has quite the opposite effect in the production of nitrogenated molecule $\mathrm{C}_{3} \mathrm{H}_{3} \mathrm{~N}^{+}$. $40 \%$ of the trimer trajectories produce $\mathrm{C}_{3} \mathrm{H}_{3} \mathrm{~N}^{+}$, while just about $30 \%$ of the tetramer and pentamer trajectories 
produce it. Having more $\mathrm{HCCH}$ helps create molecules as more trajectories actually end up in covalently bonded structures rather than ending up as clusters. A few of the trajectories (less than $10 \%$ ) of trimer, tetramer, and pentamer produce a covalently bonded $\mathrm{C}_{5} \mathrm{H}_{5} \mathrm{~N}^{+}$molecule, which is pyrimidine cation. Tetramer and pentamers also produce benzene cation $\mathrm{C}_{6} \mathrm{H}_{6}{ }^{+}$in small numbers similar to what was seen in the case of $2 \mathrm{HCN}: x \mathrm{HCCH}$ clusters.

As shown in Figures 10 and 12, the pentamer clusters can result in covalently bonded core structures that contain four of the molecules in the starting clusters. The results suggest that for even larger clusters we might end up with even larger bonded structures, and with a higher percentage of large bonded cyclic molecules, and especially cyclic organics with nitrogen in the ring. But having higher proportion of $\mathrm{HCN}$ compared to $\mathrm{HCCH}$ may not help in making larger molecules as seen from the pure $\mathrm{HCN}$ cluster calculations. Some $\mathrm{HCN}$ with a larger proportion of $\mathrm{HCCH}$ as the starting point may be the recipe for larger molecular synthesis as $\mathrm{HCCH}$ acts as the glue that grows the carbon framework. This also means that making cyclic molecules with more than one nitrogen in the ring may be rather difficult.

The different isomers resulting from the simulations are shown in Figure $13 . \mathrm{C}_{3} \mathrm{H}_{3} \mathrm{~N}^{+}$is one of the major products of association for trimer, tetramer, and pentamer cases. This structure is a direct association of $\mathrm{HCCH}^{+}$and $\mathrm{HCN}$ to produce $\mathrm{HCCHNCH}^{+}$, which is a higher energy structural isomer of vinyl cyanide cation $\left(\mathrm{H}_{2} \mathrm{CCHCN}^{+}\right)$. This isomer $\left(\mathrm{HCCHNCH}^{+}\right)$has been previously shown to be structurally important, ${ }^{61}$ as it has a CCNC framework, and another addition of HCN to it leads to pyrimidine $\left(\mathrm{C}_{4} \mathrm{~N}_{2} \mathrm{H}_{4}{ }^{+}\right)$. Conversely, dissociation of pyrimidine in an ionizing medium leads to $\mathrm{C}_{3} \mathrm{H}_{3} \mathrm{~N}^{+}{ }^{62}$ It appears from these trajectory calculations that this isomer $\left(\mathrm{HCCHNCH}^{+}\right)$ resides in a deep enough potential well to be dynamically stable on the $\mathrm{C}_{3} \mathrm{H}_{3} \mathrm{~N}^{+}$potential energy surface. We do not, however, observe any $\mathrm{C}_{4} \mathrm{~N}_{2} \mathrm{H}_{4}{ }^{+}$(i.e. addition of another $\mathrm{HCN}$ ) products from our simulations. This could be due to the presence of more reactive HCCH around. Further simulations are therefore necessary to draw firm conclusions about the possibility or impossibility of pyrimidine formation. Pyrimidine, of course, is an astrobiologically significant prebiotic molecule, and its gas phase synthesis has been proposed to go via this route. ${ }^{63}$ 


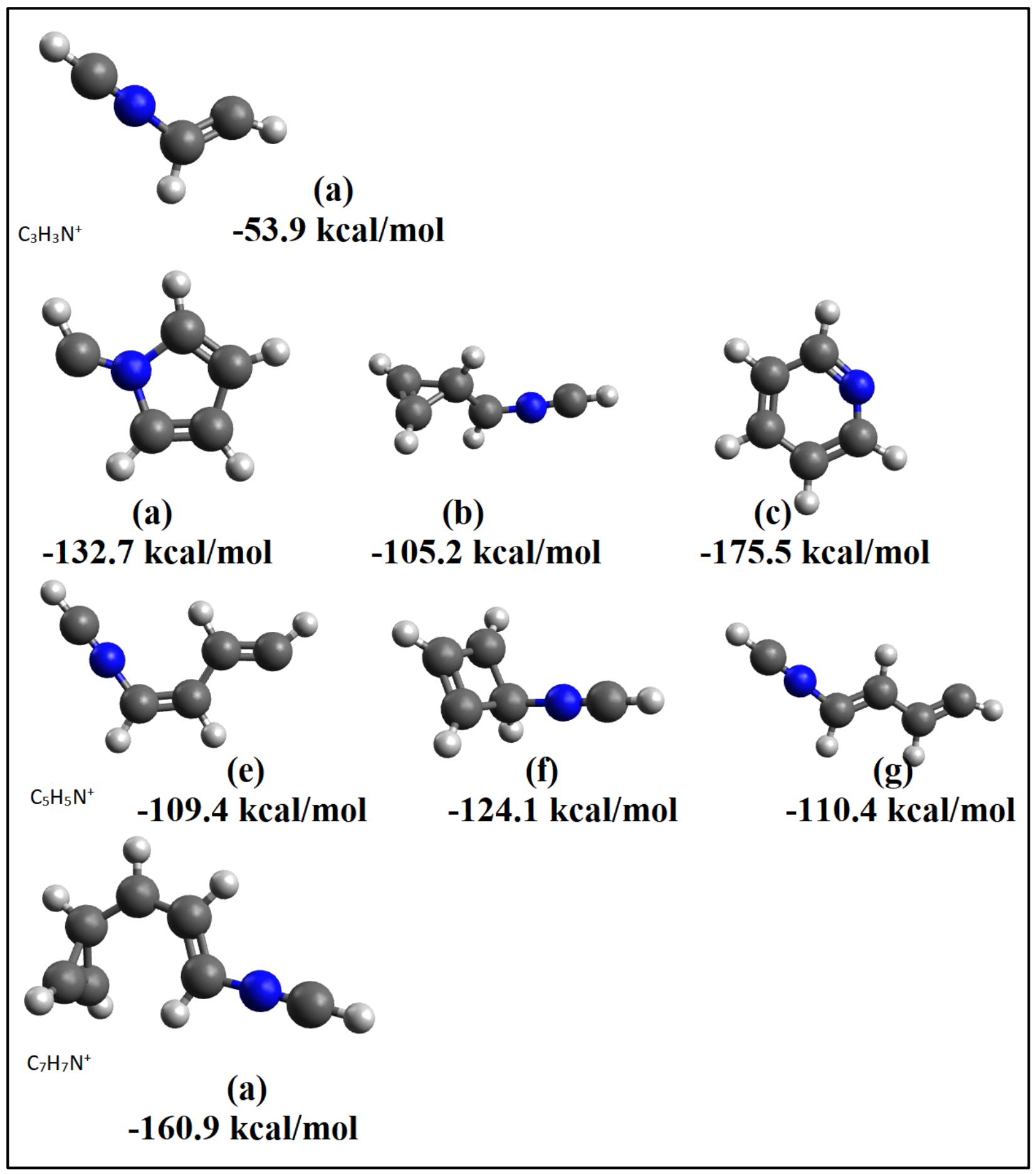

Figure 13: Different cores structures (after re-optimization) obtained in the MD simulations starting from ionization at optimized neutral $(\mathrm{HCN})_{1}\left(\mathrm{C}_{2} \mathrm{H}_{2}\right)_{x}(x=2,3,4)$ complexes. Binding energies are calculated with respect to the building blocks: $\mathrm{HCN}$ and $\mathrm{HCCH}^{+}$for $\mathrm{C}_{3} \mathrm{H}_{3} \mathrm{~N}^{+}$structure, $\mathrm{HCN}, \mathrm{HCCH}$ and $\mathrm{HCCH}^{+}$for the case of $\mathrm{C}_{5} \mathrm{H}_{5} \mathrm{~N}^{+}$structures and $\mathrm{HCN}$, two HCCH and $\mathrm{HCCH}^{+}$for the case of $\mathrm{C}_{7} \mathrm{H}_{7} \mathrm{~N}^{+}$structures.

III.f Reaction pathway to the pyridine radical cation. 
As discussed in the introduction, the pyridine molecule is an important species from the astrobiology perspective, as it is a molecule with a nitrogen in the ring, and on the pathway to DNA nucleobases. The AIMD results demonstrated the formation of pyridine cation from ionized clusters containing either 1 or $2 \mathrm{HCN}$ molecules, with the rest being acetylene. However, the number of trajectories reaching pyridine cation is too few to be statistically significant for comparison amongst the different ratio of $\mathrm{HCN}$ and acetylene runs.

To probe the underlying potential energy surface, Figure 14 shows a reaction coordinate for pyridine formation from pentamer clusters containing one $\mathrm{HCN}$ and four acetylene molecules as demonstrated in one of the simulations. As one can see from the figure, the path to form pyridine starts by bonding of two acetylene and one $\mathrm{HCN}$, releasing almost $100 \mathrm{kcal} / \mathrm{mol}$ of kinetic energy. This is followed by rearrangement to form a cyclic structure. The first transition state is very shallow $(0.39 \mathrm{kcal} / \mathrm{mol})$ followed by another for ring closure $(15.52 \mathrm{kcal} / \mathrm{mol})$. Relative to ionized reactants, these barriers are deeply submerged, meaning the internal kinetic energy of the system is much larger than these barriers. It is hence a matter of time (competing against evaporation of other molecules) before the system undergoes unimolecular rearrangement to form the far more stable cyclic pyridine radical cation. We note that the energies given here depend on the orientation of the two spectator acetylene molecules (i.e. there are multiple minima and multiple TSs). 


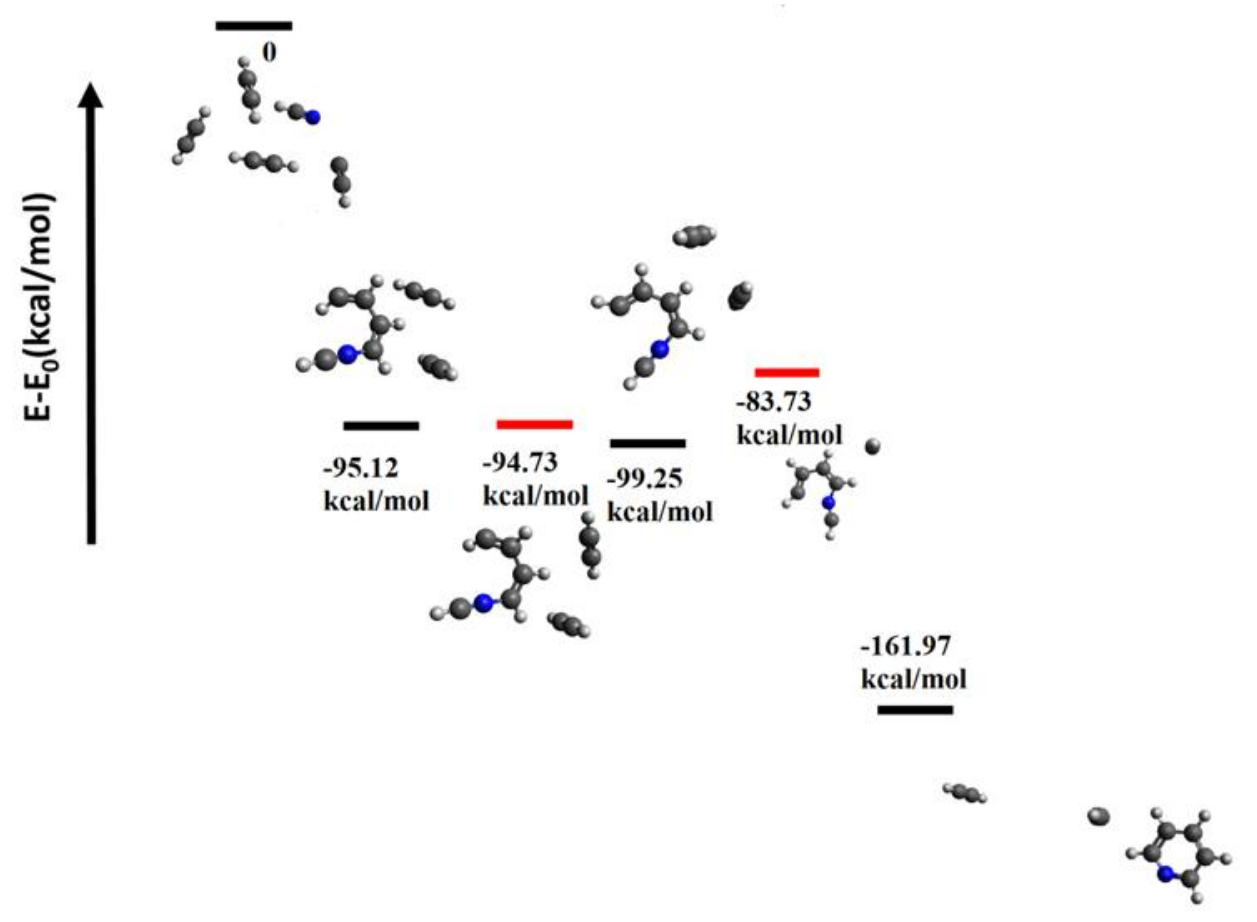

Figure 14: One pathway for pyridine formation starting from an $\mathrm{HCN}\left(\mathrm{C}_{2} \mathrm{H}_{2}\right)_{4}$ cluster geometry (on the cationic surface). Relative energies are given in $\mathrm{kcal} / \mathrm{mol}$ with respect to the cluster geometry (ionized). Black lines refer to minimum energy structures and red to transition states.

By far the most prevalent products after the trajectories complete are the reaction between $\mathrm{HCCH}$ and $\mathrm{HCN}$ leading to linear $\mathrm{C}_{3} \mathrm{H}_{3} \mathrm{~N}^{+}$, or a reaction of two acetylene units leading to cyclic $\mathrm{C}_{4} \mathrm{H}_{4}{ }^{+}$. Although in relatively low percentages, the cyclic isomers with six and seven membered rings with nitrogen in the ring also form. Which isomer of the product forms at the end of a trajectory run depends on the initial geometry from where the trajectory was launched; i.e. the geometry of the neutral cluster. The trimer clusters make primarily linear covalently bonded cationic products, while the larger clusters (tetramers and pentamers) make slightly greater percentages of cyclic products. The cyclic isomers once made in a trajectory run seem to be robust, especially for the larger tetramer and pentamer clusters. In the tetramer or pentamer cluster, the formation of cyclic isomers is helped by the evaporation of extra spectator acetylene or $\mathrm{HCN}$ molecules that take away some of the excess internal energy of the stable products, thereby cooling the cyclic products. 


\section{Conclusions}

In this manuscript, we have demonstrated that molecular growth can occur upon ionization of van der Waals clusters containing varying numbers of $\mathrm{HCN}$ and acetylene molecules. In pure HCN clusters, growth appears to be quenched at the level of pairwise condensation: the resulting products seem to be stable against further reaction in our trajectories, which can be understood chemically. We find two interesting classes of products. First, distonic products where a weakly bound $\mathrm{CN}$ radical is formed whilst protonated $\mathrm{HCNH}^{+}$forms a strong ionic hydrogen bond with an additional $\mathrm{HCN}$ molecule yielding $\mathrm{HCNH}^{+}-\mathrm{NCH}$ as a stable adduct. Second, we find a bonded $\mathrm{C}_{2} \mathrm{H}_{2} \mathrm{~N}_{2}{ }^{+}$structure, which has a very large binding energy of $-78.24 \mathrm{kcal} / \mathrm{mol}$ and contains a radical on the central nitrogen atom. This structure is a high energy isomer, and also forms very stable adducts with spectator HCN molecules as demonstrated by the large binding energies in the cluster, which can explain why we do not see further growth.

Our results show that for growth beyond 4 heavy atoms to occur, HCN clusters must be a dilute additive to clusters of acetylene molecules. In a mixed cluster of acetylene and HCN, the acetylene is the preferentially ionized monomer. Our results indicate the ionized acetylene is more effective in attacking neighboring molecules and leads to growth of larger structures including the six-membered ring compounds $\mathrm{C}_{6} \mathrm{H}_{6}{ }^{+}$and $\mathrm{C}_{5} \mathrm{H}_{5} \mathrm{~N}^{+}$when starting with the neutral pentamer clusters. Additionally, we make three key observations from the results. (i) Nitrogen addition to carbon framework via ionization of neutral clusters of $\mathrm{HCCH}$ and $\mathrm{HCN}$ is facile. (ii) Both pure and nitrogenated carbocyclic rings and chain molecules are obtained by this process as evidenced by the results. (iii) A single nitrogen atom incorporation to cyclic hydrocarbons is effective, but the incorporation of more than one nitrogen atoms in a cyclic molecule was not seen in our results (within the timeframe of our simulations) although di- and tri-nitrogenated chain molecules were observed.

Both $\mathrm{HCN}$ and $\mathrm{HCCH}$ are widespread in the interstellar medium as well as in the atmosphere of Titan. The results presented here provide a feasible pathway for molecular growth in the gas phase in cold dense molecular clouds and in Titan's upper atmosphere where the number density is relatively large, and neutral clusters may form and be ionized by radiation. We have demonstrated that pyridine, which is a nitrogen-substituted aromatic ring (in its cationic form), can be formed upon ionization of the mixed neutral clusters of $\mathrm{HCCH}$ and $\mathrm{HCN}$. We did not observe the formation of pyrimidine or diazine cation, which, may be possible starting from the 
photoionization of larger clusters. It would be very interesting to see if the predictions of these simulation studies can be verified experimentally in the future, which seems possible at least in principle using photoionization in combination with mass spectrometry, similar to successful experiments on acetylene clusters. ${ }^{44,45}$

\section{Acknowledgements}

This work was supported by the National Aeronautics and Space Administration through the NASA Astrobiology Institute under Cooperative Agreement Notice NNH13ZDA017C issued through the Science Mission Directorate.

Statement on conflicts of interest: MHG is a part-owner of Q-Chem Inc. 


\section{References}

1. Ehrenfreund, P.; Sephton, M. A., Carbon molecules in space: from astrochemistry to astrobiology. Faraday Discussions 2006, 133 (0), 277-288.

2. Rhee, Y. M.; Lee, T. J.; Gudipati, M. S.; Allamandola, L. J.; Head-Gordon, M., Charged polycyclic aromatic hydrocarbon clusters and the galactic extended red emission. Proceedings of the National Academy of Sciences of the United States of America 2007, 104 (13), 5274-5278.

3. Joblin, C.; Mulas, G., Interstellar polycylic aromatic hydrocarbons: from space to the laboratory. Eas Publications 2009, 35, 133-152.

4. Leger, A.; Puget, J. L., Identification of the 'unidentified' IR emission features of interstellar dust? Astronomy and Astrophysics 1984, 137 (1), L5 - L8.

5. Allamandola, L. J.; Tielens, A. G. G. M.; Barker, J. R., Interstellar polycyclic aromatic hydrocarbons - The infrared emission bands, the excitation/emission mechanism, and the astrophysical implications. Astrophysical Journal Supplement Series 1989, 71, 733-775.

6. Tielens, A. G. G. M., Interstellar Polycyclic Aromatic Hydrocarbon Molecules. Annual Review of Astronomy and Astrophysics 2008, 46 (1), 289-337.

7. Sellgrem, K., The near-infrared continuum emission of visual reflection nebulae. Astrophysical Journal, Part 1 1984, 277, 623-633.

8. Schmitt-Kopplin, P.; Gabelica, Z.; Gougeon, R. D.; Fekete, A.; Kanawati, B.; Harir, M.; Gebefuegi, I.; Eckel, G.; Hertkorn, N., High molecular diversity of extraterrestrial organic matter in Murchison meteorite revealed 40 years after its fall. Proceedings of the National Academy of Sciences 2010, 107 (7), 2763-2768.

9. Smith, K. E.; Callahan, M. P.; Gerakines, P. A.; Dworkin, J. P.; House, C. H., Investigation of pyridine carboxylic acids in $\mathrm{CM} 2$ carbonaceous chondrites: Potential precursor molecules for ancient coenzymes. Geochimica et Cosmochimica Acta 2014, 136, 1-12.

10. Callahan, M. P.; Smith, K. E.; Cleaves, H. J.; Ruzicka, J.; Stern, J. C.; Glavin, D. P.; House, C. H.; Dworkin, J. P., Carbonaceous meteorites contain a wide range of extraterrestrial nucleobases. Proceedings of the National Academy of Sciences 2011, 108 (34), 13995-13998.

11. Kvenvolden, K.; Lawless, J.; Pering, K.; Peterson, E.; Flores, J.; Ponnamperuma, C.; Kaplan, I. R.; Moore, C., Evidence for Extraterrestrial Amino-acids and Hydrocarbons in the Murchison Meteorite. Nature 1970, 228 (5275), 923-926.

12. Mullie, F.; Reisse, J. In Organic matter in carbonaceous chondrites, Berlin, Heidelberg, Springer Berlin Heidelberg: Berlin, Heidelberg, 1987; pp 83-117.

13. Cooper, G.; Kimmich, N.; Belisle, W.; Sarinana, J.; Brabham, K.; Garrel, L., Carbonaceous meteorites as a source of sugar-related organic compounds for the early Earth. Nature 2001, 414 (6866), 879-883.

14. Hayatsu, R.; Studier, M. H.; Moore, L. P.; Anders, E., Purines and triazines in the Murchison meteorite. Geochimica et Cosmochimica Acta 1975, 39 (4), 471-488. 
15. Martins, Z.; Botta, O.; Fogel, M. L.; Sephton, M. A.; Glavin, D. P.; Watson, J. S.; Dworkin, J. P.; Schwartz, A. W.; Ehrenfreund, P., Extraterrestrial nucleobases in the Murchison meteorite. Earth and Planetary Science Letters 2008, 270 (1), 130-136.

16. Charnley, S. B.; Kuan, Y.-J.; Huang, H.-C.; Botta, O.; Butner, H. M.; Cox, N.; Despois, D.; Ehrenfreund, P.; Kisiel, Z.; Lee, Y.-Y.; Markwick, A. J.; Peeters, Z.; Rodgers, S. D., Astronomical searches for nitrogen heterocycles. Advances in Space Research 2005, 36 (2), 137-145.

17. Kuan, Y.-J.; Charnley, S. B.; Huang, H.-C.; Kisiel, Z.; Ehrenfreund, P.; Tseng, W.-L.; Yan, C.-H., Searches for interstellar molecules of potential prebiotic importance. Advances in Space Research 2004, 33 (1), 31-39.

18. Croiset, B. A.; Candian, A.; Berné, O.; Tielens, A. G. G. M., Mapping PAH sizes in NGC 7023 with SOFIA. A\&A 2016, 590, A26.

19. Berné, O.; Montillaud, J.; Joblin, C., Top-down formation of fullerenes in the interstellar medium. Astronomy and Astrophysics 2015, 577, A133.

20. Allamandola, L. J.; Tielens, A. G. G. M.; Barker, J. R., Polycyclic aromatic hydrocarbons and the unidentified infrared emission bands: auto exhaust along the milky way. The Astrophysical Journal 1985, 290, L25.

21. McGuire, B. A.; Burkhardt, A. M.; Kalenskii, S.; Shingledecker, C. N.; Remijan, A. J.; Herbst, E.; McCarthy, M. C., Detection of the aromatic molecule benzonitrile (\&lt;em\&gt;c\&lt;/em\&gt;-C\&lt;sub\&gt;6\&lt;/sub\&gt;H\&lt;sub\&gt;5\&lt;/sub\&gt;CN) in the interstellar medium. Science 2018, 359 (6372), 202.

22. Chyba, C.; Thomas, P.; Brookshaw, L.; Sagan, C., Cometary delivery of organic molecules to the early Earth. Science 1990, 249 (4967), 366-373.

23. Burton, A. S.; Stern, J. C.; Elsila, J. E.; Glavin, D. P.; Dworkin, J. P., Understanding prebiotic chemistry through the analysis of extraterrestrial amino acids and nucleobases in meteorites. Chemical Society Reviews 2012, 41 (16), 5459-5472.

24. Sandford, S. A.; Nuevo, M.; Bera, P. P.; Lee, T. J., Prebiotic Astrochemistry and the Formation of Molecules of Astrobiological Interest in Interstellar Clouds and Protostellar Disks. Chemical Reviews 2020.

25. Belloche, A.; Garrod, R. T.; Müller, H. S. P.; Menten, K. M.; Comito, C.; Schilke, P., Increased complexity in interstellar chemistry: detection and chemical modeling of ethyl formate and n-propyl cyanide in Sagittarius B2(N) ***. A\&A 2009, 499 (1), 215-232.

26. Pagani, L.; Favre, C.; Goldsmith, P. F.; Bergin, E. A.; Snell, R.; Melnick, G., The complexity of Orion: an ALMA view. A\&A 2017, 604, A32.

27. Johnson, D. R.; Lovas, F. J.; Gottlieb, C. A.; Gottlieb, E. W.; Litvak, M. M.; Guelin, M.; Thaddeus, P., Detection of interstellar ethyl cyanide. The Astrophysical Journal 1977, 218, 370376.

28. Cazaux, S.; Tielens, A. G. G. M.; Ceccarelli, C.; Castets, A.; Wakelam, V.; Caux, E.; Parise, B.; Teyssier, D., The Hot Core around the Low-Mass Protostar IRAS 16293-2422: Scoundrels Rule! The Astrophysical Journal 2003, 593 (1), L51-L55.

29. Kroto, H. W.; Kirby, C.; Walton, D. R. M.; Avery, L. W.; Broten, N. W.; MacLeod, J. M.; Oka, T., The detection of cyanohexatriyne, $\mathrm{H}(\mathrm{C} \equiv \mathrm{C})<\mathrm{SUB}>3</ \mathrm{SUB}>\mathrm{CN}$, in Heile's Cloud 2. The Astrophysical Journal 1978, 219, L133-L137.

30. Bera, P. P.; Head-Gordon, M.; Lee, T. J., Initiating molecular growth in the interstellar medium via dimeric complexes of observed ions and molecules . A\&A 2011, 535, A74. 
31. Bera, P. P.; Peverati, R.; Head-Gordon, M.; Lee, T. J., Hydrocarbon growth via ionmolecule reactions: computational studies of the isomers of $\mathrm{C} 4 \mathrm{H} 2+, \mathrm{C} 6 \mathrm{H} 2+$ and $\mathrm{C} 6 \mathrm{H} 4+$ and their formation paths from acetylene and its fragments. Physical Chemistry Chemical Physics 2015, 17 (3), 1859-1869.

32. Bera, P. P.; Head-Gordon, M.; Lee, T. J., Association mechanisms of unsaturated C2 hydrocarbons with their cations: acetylene and ethylene. Physical Chemistry Chemical Physics 2013, 15 (6), 2012-2023.

33. Parker, D. S. N.; Kaiser, R. I.; Kostko, O.; Troy, T. P.; Ahmed, M.; Mebel, A. M.; Tielens, A. G. G. M., Gas Phase Synthesis of (Iso)Quinoline and Its Role in the Formation of Nucleobases in the Interstellar Medium. The Astrophysical Journal 2015, 803 (2), 53.

34. Ziurys, L. M., The chemistry in circumstellar envelopes of evolved stars: Following the origin of the elements to the origin of life. Proceedings of the National Academy of Sciences 2006, 103 (33), 12274-12279.

35. Parker, D. S. N.; Yang, T.; Dangi, B. B.; Kaiser, R. I.; Bera, P. P.; Lee, T. J., Low Temperature Formation of Nitrogen-substituted Polycyclic Aromatic Hydrocarbons (PANHs)- Barrierless Routes to Dihydro(iso)quinolines. The Astrophysical Journal 2015, 815 (2), 115.

36. Barks, H. L.; Buckley, R.; Grieves, G. A.; Di Mauro, E.; Hud, N. V.; Orlando, T. M., Guanine, Adenine, and Hypoxanthine Production in UV-Irradiated Formamide Solutions: Relaxation of the Requirements for Prebiotic Purine Nucleobase Formation. ChemBioChem 2010, 11 (9), 1240-1243.

37. Matthews, C. N.; Ludicky, R., Hydrogen cyanide polymers on comets. Advances in Space Research 1992, 12 (4), 21-32.

38. Matthews, C. N.; Minard, R. D., Hydrogen cyanide polymers, comets and the origin of life. Faraday Discussions 2006, 133 (0), 393-401.

39. Matthews, C. N., Heteropolypeptides on Titan? Origins of life 1982, 12 (3), 281-283.

40. Woeller, F.; Ponnamperuma, C., Organic synthesis in a simulated Jovian atmosphere. Icarus 1969, 10 (3), 386-392.

41. Shemansky, D. E.; Stewart, A. I. F.; West, R. A.; Esposito, L. W.; Hallett, J. T.; Liu, X., The Cassini UVIS Stellar Probe of the Titan Atmosphere. Science 2005, 308 (5724), 978-982.

42. Waite, J. H.; Niemann, H.; Yelle, R. V.; Kasprzak, W. T.; Cravens, T. E.; Luhmann, J. G.; McNutt, R. L.; Ip, W.-H.; Gell, D.; De La Haye, V.; Müller-Wordag, I.; Magee, B.; Borggren, N.; Ledvina, S.; Fletcher, G.; Walter, E.; Miller, R.; Scherer, S.; Thorpe, R.; Xu, J.; Block, B.; Arnett, K., Ion Neutral Mass Spectrometer Results from the First Flyby of Titan. Science 2005, 308 (5724), 982-986.

43. Bandyopadhyay, B.; Stein, T.; Fang, Y.; Kostko, O.; White, A.; Head-Gordon, M.; Ahmed, M., Probing ionic complexes of ethylene and acetylene with vacuum-ultraviolet radiation. The Journal of Physical Chemistry A 2016, 120 (27), 5053-5064.

44. Ahmed, M.; Kostko, O., From atoms to aerosols: probing clusters and nanoparticles with synchrotron based mass spectrometry and X-ray spectroscopy. Physical Chemistry Chemical Physics 2020, 22 (5), 2713-2737.

45. Stein, T.; Bandyopadhyay, B.; Troy, T. P.; Fang, Y.; Kostko, O.; Ahmed, M.; Head-Gordon, M., Ab initio dynamics and photoionization mass spectrometry reveal ion-molecule pathways from ionized acetylene clusters to benzene cation. Proc Natl Acad Sci U S A 2017, 114 (21), E4125E4133. 
46. Ridgway, S. T.; Hall, D. N. B.; Kleinmann, S. G.; Weinberger, D. A.; Wojslaw, R. S., Circumstellar acetylene in the infrared spectrum of IRC $+10^{\circ} 216$. Nature 1976, 264 (5584), 345346.

47. Lacy, J. H.; Evans, N. J., II; Achtermann, J. M.; Bruce, D. E.; Arens, J. F.; Carr, J. S., Discovery of Interstellar Acetylene. The Astrophysical Journal 1989, 342, L43.

48. Cernicharo, J.; Heras, A. M.; Tielens, A. G. G. M.; Pardo, J. R.; Herpin, F.; Guélin, M.; Waters, L. B. F. M., Infrared Space Observatory's Discovery of $\mathrm{C} 4 \mathrm{H} 2, \mathrm{C} 6 \mathrm{H} 2$, and Benzene in $\mathrm{CRL}$ 618. The Astrophysical Journal 2001, 546 (2), L123-L126.

49. Stein, T.; Jose, J., Molecular Formation upon Ionization of van der Waals Clusters and Implication to Astrochemistry. Israel Journal of Chemistry 2020, 60, 1-9.

50. Prasad, S. S.; Tarafdar, S. P., UV radiation field inside dense clouds - Its possible existence and chemical implications. The Astrophysical Journal 1983, 267, 603-609.

51. Ruaud, M.; Gorti, U., A Three-phase Approach to Grain Surface Chemistry in Protoplanetary Disks: Gas, Ice Surfaces, and Ice Mantles of Dust Grains. The Astrophysical Journal 2019, 885 (2), 146.

52. Hörst, S. M., Titan's atmosphere and climate. Journal of Geophysical Research: Planets 2017, 122 (3), 432-482.

53. Huang, C.; Zhang, F.; Kaiser, R. I.; Kislov, V. V.; Mebel, A. M.; Silva, R.; Gichuhi, W. K.; Suits, A. G., PHOTODISSOCIATION OF THE DIACETYLENE DIMER AND IMPLICATIONS FOR HYDROCARBON GROWTH IN TITAN'S ATMOSPHERE. The Astrophysical Journal 2010, 714 (2), 1249-1255.

54. Shao, Y.; Gan, Z.; Epifanovsky, E.; Gilbert, A. T. B.; Wormit, M.; Kussmann, J.; Lange, A. W.; Behn, A.; Deng, J.; Feng, X.; Ghosh, D.; Goldey, M.; Horn, P. R.; Jacobson, L. D.; Kaliman, I.; Khaliullin, R. Z.; Kuś, T.; Landau, A.; Liu, J.; Proynov, E. I.; Rhee, Y. M.; Richard, R. M.; Rohrdanz, M. A.; Steele, R. P.; Sundstrom, E. J.; Woodcock, H. L.; Zimmerman, P. M.; Zuev, D.; Albrecht, B.; Alguire, E.; Austin, B.; Beran, G. J. O.; Bernard, Y. A.; Berquist, E.; Brandhorst, K.; Bravaya, K. B.; Brown, S. T.; Casanova, D.; Chang, C.-M.; Chen, Y.; Chien, S. H.; Closser, K. D.; Crittenden, D. L.; Diedenhofen, M.; DiStasio, R. A.; Do, H.; Dutoi, A. D.; Edgar, R. G.; Fatehi, S.; Fusti-Molnar, L.; Ghysels, A.; Golubeva-Zadorozhnaya, A.; Gomes, J.; Hanson-Heine, M. W. D.; Harbach, P. H. P.; Hauser, A. W.; Hohenstein, E. G.; Holden, Z. C.; Jagau, T.-C.; Ji, H.; Kaduk, B.; Khistyaev, K.; Kim, J.; Kim, J.; King, R. A.; Klunzinger, P.; Kosenkov, D.; Kowalczyk, T.; Krauter, C. M.; Lao, K. U.; Laurent, A. D.; Lawler, K. V.; Levchenko, S. V.; Lin, C. Y.; Liu, F.; Livshits, E.; Lochan, R. C.; Luenser, A.; Manohar, P.; Manzer, S. F.; Mao, S.-P.; Mardirossian, N.; Marenich, A. V.; Maurer, S. A.; Mayhall, N. J.; Neuscamman, E.; Oana, C. M.; Olivares-Amaya, R.; O'Neill, D. P.; Parkhill, J. A.; Perrine, T. M.; Peverati, R.; Prociuk, A.; Rehn, D. R.; Rosta, E.; Russ, N. J.; Sharada, S. M.; Sharma, S.; Small, D. W.; Sodt, A.; Stein, T.; Stück, D.; Su, Y.-C.; Thom, A. J. W.; Tsuchimochi, T.; Vanovschi, V.; Vogt, L.; Vydrov, O.; Wang, T.; Watson, M. A.; Wenzel, J.; White, A.; Williams, C. F.; Yang, J.; Yeganeh, S.; Yost, S. R.; You, Z.-Q.; Zhang, I. Y.; Zhang, X.; Zhao, Y.; Brooks, B. R.; Chan, G. K. L.; Chipman, D. M.; Cramer, C. J.; Goddard, W. A.; Gordon, M. S.; Hehre, W. J.; Klamt, A.; Schaefer, H. F.; Schmidt, M. W.; Sherrill, C. D.; Truhlar, D. G.; Warshel, A.; Xu, X.; Aspuru-Guzik, A.; Baer, R.; Bell, A. T.; Besley, N. A.; Chai, J.-D.; Dreuw, A.; Dunietz, B. D.; Furlani, T. R.; Gwaltney, S. R.; Hsu, C.-P.; Jung, Y.; Kong, J.; Lambrecht, D. S.; Liang, W.; Ochsenfeld, C.; Rassolov, V. A.; Slipchenko, L. V.; Subotnik, J. E.; Van Voorhis, T.; Herbert, J. M.; Krylov, A. I.; Gill, P. M. W.; Head-Gordon, M., Advances in molecular quantum 
chemistry contained in the Q-Chem 4 program package. Molecular Physics 2015, 113 (2), 184215.

55. Mardirossian, N.; Head-Gordon, M., [small omega]B97X-V: A 10-parameter, rangeseparated hybrid, generalized gradient approximation density functional with nonlocal correlation, designed by a survival-of-the-fittest strategy. Physical Chemistry Chemical Physics 2014, 16 (21), 9904-9924.

56. Dunning , T. H., Jr, Gaussian basis sets for use in correlated molecular calculations. I. The atoms boron through neon and hydrogen. The Journal of Chemical Physics 1989, 90 (2), 10071023.

57. Vydrov, O. A.; Van Voorhis, T., Nonlocal van der Waals density functional: The simpler the better. The Journal of Chemical Physics 2010, 133 (24), 244103.

58. Goerigk, L.; Hansen, A.; Bauer, C.; Ehrlich, S.; Najibi, A.; Grimme, S., A look at the density functional theory zoo with the advanced GMTKN55 database for general main group thermochemistry, kinetics and noncovalent interactions. Physical Chemistry Chemical Physics 2017, 19 (48), 32184-32215.

59. Mardirossian, N.; Head-Gordon, M., Thirty years of density functional theory in computational chemistry: an overview and extensive assessment of 200 density functionals. Molecular Physics 2017, 115 (19), 2315-2372.

60. Chai, J.-D.; Head-Gordon, M., Systematic optimization of long-range corrected hybrid density functionals. The Journal of Chemical Physics 2008, 128 (8), 084106.

61. Bera, P. P.; Lee, T. J.; Schaefer , H. F., III, Are isomers of the vinyl cyanide ion missing links for interstellar pyrimidine formation? The Journal of Chemical Physics 2009, 131 (7), 074303.

62. Fondren, L. D.; McLain, J.; Jackson, D. M.; Adams, N. G.; Babcock, L. M., Studies of reactions of a series of ions with nitrogen containing heterocyclic molecules using a selected ion flow tube. International Journal of Mass Spectrometry 2007, 265 (1), 60-67.

63. Hamid, A. M.; Bera, P. P.; Lee, T. J.; Aziz, S. G.; Alyoubi, A. O.; El-Shall, M. S., Evidence for the Formation of Pyrimidine Cations from the Sequential Reactions of Hydrogen Cyanide with the Acetylene Radical Cation. The Journal of Physical Chemistry Letters 2014, 5 (19), 3392-3398. 